\title{
BOUNDED POLYMEASURES AND ASSOCIATED TRANSLATION COMMUTATIVE POLYNOMIAL OPERATORS
}

\author{
DAVID R. BRILLINGER
}

1. Introduction. Morse and Transue [6-8] defined and developed properties of bimeasures, quadratic generalizations of the linear Radon measures. This paper is devoted to a polynomial generalization in the bounded case, the polymeasure, and to indicating that bounded polymeasures provide a means of representing translation commutative polynomial operators on the space $C(G)$ of bounded continuous functions on a locally compact group $G$ when the operators are strictly bounded and strictly continuous on strictly bounded sets. In the linear case the result demonstrated is; a left translation invariant operator on $C(G)$ takes the form $y(h)=\int x(h g) d \Lambda(g)$ with $\Lambda$ a bounded Radon measure, $x(g), y(g) \in C(G)$, if and only if the operator is strictly bounded and strictly continuous on strictly bounded sets. The desire to obtain a representation for translation invariant polynomial operators on function space results from a growing use of such operators in stochastic process theory, see [5], [9].

2. Bounded polymeasures. Throughout the paper bold face letters $\boldsymbol{f}, \boldsymbol{m}, \boldsymbol{p}$ will denote $k$-vectors. If $X$ is locally compact $C(X)$ will denote the set of bounded, complex-valued, continuous functions on $X$, $C_{0}(X)$ will denote the $f$ in $C(X)$ which vanish at infinity, $C_{00}(X)$ will denote the $f$ in $C(X)$ with compact support. If there is no confusion as to the basic space $X$ we will write $C, C_{0}, C_{00}$. For real-valued functions $f, g$ on a set $X$, the expression $f \leqq g$ will mean $f(x) \leqq g(x)$ for all $x$ in $X$, while for $k$-vectors $f \leqq g$ will mean $f_{i} \leqq g_{i}$ for each coordinate. If $A$ is a linear space, $A^{k}$ will denote the direct product of $A$ with itself $k$ times. When $A$ has a topology, we will consider two sorts of continuity in $A^{k}$, continuity separately in each coordinate and joint continuity in all coordinates. The norm $\|f\|$ on $C, C_{0}$, and $C_{00}$ will denote the supremum norm.

Buck [1] introduced the strict topology on $C(X)$. It is a locally convex topology defined by the family of seminorms $\|m\|_{\phi}$ $=\sup |m(x) \phi(x)|$, for $m$ in $C$ and $\phi$ in $C_{0}$. The topology is complete, its bounded sets are the bounded sets of the uniform topology and on a bounded set the topology coincides with the unitorm on compacta topology [2].

Received by the editors March 4, 1966. 
DEFINITION. A bounded $k$ th-order polymeasure is a uniformly continuous, multilinear, complex-valued functional on $C_{00}(X)^{k}$ where $X$ is a locally compact space. Its values will be denoted by $\Lambda(f)$ where $f$ is in $C_{00}^{\mathbf{k}}$. The uniform bound will be denoted $\|\Lambda\|$.

We note that if $k=1, \Lambda$ is the usual Radon integral, while if $k=2$, $\Lambda$ is the $C$-bimeasure considered in [6-8].

Suppose $p \geqq 0$ is in $C(X)^{k}$. We define, for $\Lambda$ a bounded polymeasure,

$$
\Lambda^{*}(p)=\sup _{|f| \leqq p}|\Lambda(f)|
$$

for $f$ in $C_{00}^{\mathbf{k}}$. By elementary calculations we see that,

(a) $\Lambda^{*}(p) \leqq \Lambda^{*}(q)$ if $p \leqq q$,

(b) $\Lambda^{*}\left(a_{1} p_{1}, \cdots, a_{k} p_{k}\right)=a_{1} \cdots a_{k} \Lambda^{*}\left(p_{1}, \cdots, p_{k}\right)$ for real $a_{j} \geqq 0$,

(c) $\Lambda^{*}\left(p_{1}+q_{1}, p_{2}, \cdots, p_{k}\right) \leqq \Lambda^{*}\left(p_{1}, \cdots, p_{k}\right)+\Lambda^{*}\left(q_{1}, p_{2}, \cdots, p_{k}\right)$ with a similar result for the second, third, . . arguments of $\Lambda^{*}, q_{1} \geqq 0$,

(d) $\Lambda^{*}(p) \leqq\|\Lambda\|\left\|p_{1}\right\| \cdots\left\|p_{k}\right\|$

(e) $\Lambda^{*}(1)=\|\Lambda\|$.

Let us now proceed to an extension of $\Lambda$ to $C^{k}$.

Theorem 1. A bounded kth-order polymeasure $\Lambda$ on $C_{00}^{\mathbf{k}}$ has a unique extension $\bar{\Lambda}$ to $C^{k}$ such that

(i) $\bar{\Lambda}$ is strictly continuous in each of its arguments,

(ii) $\left|\bar{\Lambda}\left(m_{1}, \cdots, m_{k}\right)\right| \leqq \Lambda^{*}\left(\left|m_{1}\right|, \cdots,\left|m_{k}\right|\right)$,

(iii) $\bar{\Lambda}$ is jointly uniformly continuous and

(iv) $\bar{\Lambda}$ is jointly strictly continuous on strictly bounded sets.

Proof. We extend $\Lambda$ by recursion. For $j=0, \cdots, k-1$ in turn, let $\Lambda_{j}$ be uniformly continuous and multilinear on $L_{j}=C^{j} \times C_{00}^{k-j}$, then $m_{j+1} \rightarrow \Lambda_{j}\left(m_{1}, \cdots, m_{j+1}, \cdots, m_{k}\right)$ is a uniformly continuous linear functional on $C_{00}$ with norm $\left\|\Lambda_{j}\right\|\left\|m_{1}\right\| \cdots\left\|m_{j}\right\|\left\|m_{j+2}\right\| \cdots\left\|m_{k}\right\|$ and is thus a Radon measure. It therefore (Buck [2]) has a unique extension to $C$ which is continuous in the strict topology and retains its bound. Thus we get a multilinear functional defined on $L_{j+1}$ with normal equal to $\left\|\Lambda_{j}\right\| . \Lambda_{k}$ is the required extension, $\bar{\Lambda}$, of $\Lambda=\Lambda_{0}$ to $C^{k}$. To see this we first note that $\bar{\Lambda}$ is strictly continuous in each of its arguments and that it does not depend on the order in which the $C_{00}$ 's are changed into $C$ 's for we may take any pair of coordinates and allow only these two to vary and we have a bimeasure and its usual extension (Theorem 11.1 of [8]).

(ii) follows from the definition of $\Lambda^{*}$ and the fact that we may evaluate $\bar{\Lambda}\left(m_{1}, \cdots, m_{k}\right)$ as

$$
\lim _{\alpha_{1}} \cdots \lim _{\alpha_{k}} \Lambda\left(m_{1 \alpha_{1}}, \cdots, m_{k \alpha_{k}}\right)
$$


where $m_{j \alpha_{j}}$ is a generalized sequence of elements of $C_{00}$ that converges to $m_{j}$ in the strict topology.

(iii) follows from (ii) and since $\Lambda^{*}\left(\left|m_{1}\right|, \cdots,\left|m_{k}\right|\right) \leqq\|\Lambda\|\left\|m_{1}\right\|$ ... $\left\|m_{k}\right\|$.

To prove (iv) we first note that an obvious extension of Theorem 6.1 of [7] indicates the existence of $e_{j}$ in $C_{00}, 0 \leqq e_{j} \leqq 1$ such that $\Lambda^{*}\left(1, \cdots, 1,1-e_{j}, 1, \cdots, 1\right)$ is as small as desired. Now consider $\left\{\boldsymbol{m}_{\alpha}\right\}_{\alpha \in \Delta}$ a uniformly bounded generalized sequence of elements of $C^{k},\left\|m_{j \alpha}\right\| \leqq M_{j}$, converging to $m$ in the joint topology of uniform convergence on compacta. $e_{j}$ has compact support; therefore there exists $\alpha_{0}$ such that for $\alpha>\alpha_{0},\left\|\left(m_{j}-m_{j \alpha}\right) e_{j}\right\|$ is as small as desired.

$$
\begin{aligned}
\mid \bar{\Lambda}(\boldsymbol{m}) & -\bar{\Lambda}\left(\boldsymbol{m}_{\alpha}\right) \mid \\
& \leqq\left|\sum \bar{\Lambda}\left(m_{1}, \cdots, m_{j-1}, m_{j}-m_{j \alpha}, m_{j+1 \alpha}, \cdots, m_{k \alpha}\right)\right| \\
& \leqq \sum \Lambda^{*}\left(M_{1}, \cdots, M_{j-1},\left|m_{j}-m_{j \alpha}\right|, M_{j+1}, \cdots, M_{k}\right) .
\end{aligned}
$$

Now $\Lambda^{*}\left(M_{1}, \cdots, M_{j-1},\left|m_{j}-m_{j \alpha}\right|, M_{j+1}, \cdots, M_{k}\right)$ is less than or equal a multiple of

$$
\left\|\left(m_{j}-m_{j \alpha}\right) e_{j}\right\|+\left\|m_{j}-m_{j \alpha}\right\| \Lambda^{*}\left(1, \cdots, 1,1-e_{j}, 1, \cdots, 1\right) .
$$

Using the uniform boundedness of the sequence $\left\{\boldsymbol{m}_{\alpha}\right\}_{\alpha \in \Delta}$ we see that (2.1) may be made as small as desired and $\bar{\Lambda}\left(\boldsymbol{m}_{j \alpha}\right) \rightarrow \bar{\Lambda}(\boldsymbol{m})$, proving (iv).

The extension $\bar{\Lambda}$ is seen to be unique and will also be called a bounded $k$ th-order polymeasure.

3. Translation commutative polynomial operators. In this section a 1-1 correspondence will be set up between bounded polymeasures on a locally compact group $G$ and $k$ th degree, left translation commutative, monomial operators on $C(G)$ which are strictly bounded and strictly continuous on strictly bounded sets.

Polynomial operators are discussed in Hille and Philips [3] and Mazur and Orlicz [4] for example. Given linear spaces $\mathfrak{X}$ and $\mathfrak{Y}$ a map $P: \mathfrak{X} \rightarrow \mathfrak{Y}$ is defined to be a polynomial of degree $k$ if

$$
\triangle_{u}^{k+1} P(x)=0
$$

for all $x, u$ in $\mathfrak{X}$ where $\Delta_{u} P(x)=P(x+u)-P(x)$ and 0 is the zero of $\mathfrak{Y}$.

A polynomial map of degree $k$ is a monomial map of degree $k$ if $M(\alpha x)=\alpha^{k} M(x)$ for all scalars $\alpha$. One may associate uniquely with each monomial map of degree $k$ a $k$ th-order multilinear map $N: \mathfrak{X}^{k} \rightarrow \mathfrak{V}$ called the polar. A polynomial map of degree $k$ may be written as the 
sum of monomials of degrees less than or equal to $k$. These details may be found in [3].

It is important to note that when $\mathfrak{X}$ and $\mathfrak{Y}$ are linear topological spaces, a monomial map $M$ is continuous if and only if its polar is continuous. (This result is Theorem 26.2.6 of [3] for Banach spaces. The needed result is a straightforward generalization.)

Henceforth considerations will be limited to left translations of elements of the group $G$. Given functions $m(g)$ on $G$ the translation operator, $U_{h}$, is defined by $U_{h} m(g)=m(h g), h$ in $G . e$ is the identity of $G$.

Theorem 2. The pair of relations (i) $\Omega(m)=M(m)(e)$, (ii) $M(m)(g)$ $\Omega\left(U_{g} m\right)$ set up a 1-1 correspondence between kth-order, left translation commutative, monomial operators $M: C(G) \rightarrow C(G)$ and kth-order monomial functions $\Omega: C(G) \rightarrow$ complex numbers, such that if $\Omega$ is strictly continuous on strictly bounded sets, then $M$ is strictly bounded and strictly continuous on strictly bounded sets and conversely.

Proof. The proof of a 1-1 correspondence and the stated continuity is identical with the corresponding proof in [1].

We conclude the proof by noting that (i) and (ii) do in fact define monomials under the stated assumptions.

Theorem 3. A kth-order monomial operator $M: C(G) \rightarrow C(G)$ is strictly bounded and strictly continuous on strictly bounded sets if and only if it has the form

$$
M(m)(g)=\Lambda\left(U_{o} m, \cdots, U_{\bullet} m\right)
$$

where $\Lambda$ is a bounded kth-order polymeasure and $m$ belongs to $C(G)$. An $M$ given by (3.1) is also continuous in the uniform topology and has as bound $\|\Lambda\|$, the bound of the polymeasure $\Lambda$.

Proof. The theorem follows directly. Theorem 2 indicates the existence of a monomial $\Omega$ with certain continuity properties. Take $\Lambda$ to be the polar of $\Omega$. The continuity properties that $\Lambda$ inherits make it a bounded polymeasure. (3.1) now follows from the relation between a monomial and its polar (see [3] for this last).

It was noted earlier that a polynomial of order $k$ may be expressed as the sum of monomials of orders less than or equal to $k$. These monomials are continuous if the polynomial is and conversely. (This results from the fact that the monomials are linear combinations of values of the polynomial.) We now see

Corollary. A kth order polynomial operator $P: C(G) \rightarrow C(G)$ is 
strictly bounded and strictly continuous on strictly bounded sets if and only if it has, for $m$ in $C(G)$, the form

$$
P(m)(g)=\sum_{j=0}^{k} \Lambda_{j}\left(U_{\diamond} m, \cdots, U_{\diamond} m\right)
$$

where $\Lambda_{j}$ is a bounded jth-order polymeasure.

\section{REFERENCES}

1. R. C. Buck, Operator algebras and dual spaces, Proc. Amer. Math. Soc. 3 (1952), 681-687.

2. - Bounded continuous functions on a locally compact space, Michigan Math. J. 5 (1958), 95-104.

3. E. Hille and R. S. Philips, Functional analysis and semi-groups, Colloq. Publ., Amer. Math. Soc., Providence, R. I., 1957.

4. S. Mazur and W. Orlicz, Grundlegende Eigenschaften der polynomischen Operationem, Studia Math. 5 (1934), 50-68, 179-189.

5. E. J. McShane, Stochastic integrals and non-linear processes, J. Math. Mech. 11 (1962), 235-284.

6. M. Morse, Bimeasures and their integral extensions, Ann. Mat. Pura Appl. 39 (1955), 345-356.

7. M. Morse and W. Transue, C-bimeasures $\Lambda$ and their superior integrals $\Lambda^{*}$, Rend. Circ. Mat. Palermo 4 (1955), 270-300.

8. - C-bimeasures and their integral extensions, Ann. of Math. 64 (1956), 480-504.

9. N. Wiener, Non-linear problems in random theory, The MIT Press, Cambridge, Mass., 1958.

The London School of Economics and Political Science 